\title{
Disclosed: quasiparticle properties and dynamics in real and
} momentum space

\author{
Ursula Wurstbauer ${ }^{1}$ \\ ${ }^{1}$ University of Münster Department of Physics
}

May 17, 2021

Disclosed: quasiparticle properties and dynamics in real and momentum space

Ursula Wurstbauer

Institute of Physics and Center for Soft Nanoscience (SoN), Westfälische Wilhelms-Universität Münster, Wilhelm-Klemm-Str.10, 48149 Münster, Germany.

\section{+ wurstbauer@uni-muenster.de}

Physical properties of solid-state materials and other systems are determined by manifold interactions. While for weakly interacting systems their description in a single-particle picture is often sufficient, this approach fails when the interactions become stronger and many particles are involved. The quantum-mechanical problem of describing elementary excitations and collective behavior in many-body systems is often solved by introducing quasiparticles, thereby transforming the problem of strongly interacting particles to that of weakly interacting quasiparticles.

Prominent examples are band-electrons in crystals that are described by electrons with an effective mass acting as free electrons in vacuum despite being surrounded by many other electrons and the atoms of the solid . Further examples are Cooper pairs - quasiparticles in superconductors - that are composite bosons comprised of two electrons coupled by exchange of virtual phonons ; composite fermions - quasiparticles of fractional quantum Hall effect states - consisting of electrons coupled with an even number of flux quanta ; and also phonons, the quasiparticles of collective lattice vibrations, or plasmons, the quasiparticles describing collective excitation of free electrons in solids . Quasiparticles are often connected with emergent phenomena such as superconductivity, superfluidity or others and can have different quasiparticle statistics than the underlying real-particles, e.g. transforming two fermions into a composite boson as, e.g., for Cooper pairs and excitons. The latter amounts to a bound electron-hole pair in a solid or a molecule.

The concept of employing weakly interacting quasiparticles to describe many-body phenomena in a solidstate system was pioneered by Y. Frenkel in 1930 when he described the neutral excitation of a solid with light by an exciton - a photo-excited electron-hole pair bound together by attractive Coulomb force. Excitons can be viewed as analogues of the hydrogen atom model, featuring Rydberg states and a fine structure, also with respect to spin alignment. Excitons exist in semiconductors, insulators and molecules, in zero-dimensional quantum dots, one-dimensional wires and tubes, two-dimensional systems as well as in bulk materials. Recently, the exciton-dominated light-matter interaction in ultimately thin two-dimensional semiconductors has been intensely studied because of the strong exciton binding energies on the order of $0.5 \mathrm{eV}$ caused by reduced screening. The most prominent ambassador of such systems is the class of air-stable transition metal dichalcogenides (TMDCs) as, e.g., $\mathrm{MoS}_{2}$ or $\mathrm{WSe}_{2}$ that transition from an indirect to a direct gap material when approaching the monolayer limit. The single-particle states for two valleys in the conduction band are very close in energy and prone to external stimuli such as strain doping or dielectric environment. Therefore, the nature of the lowest energy neutral interband excitation forming the exciton is under debate. 
The exciton could hold vanishing crystal momentum or finite crystal momentum such that the decay is typically phonon-assisted in order to fulfill the momentum conservation law . The all-surface nature of those materials allows modifying the excitonic properties by dielectric engineering, defect engineering by creating individual emitter sites as well stacking into van der Waals heterostructures enabling the formation of charge transfer excitons (a.k.a interlayer excitons) with one electron localized in one crystal plane and another electron in the other plane .

Excitons are electrically neutral and can be generated electrically or optically and are either localized around an individual lattice site (Frenkel exciton) or freely moving in a crystal (Wannier-Mott exciton) . Excitons can act as single photon sources emitting just one exciton at a time or gather into dense exciton ensembles. Excitons play also a crucial role in the origin of life as energy transfer mechanisms in biology via Forster resonance energy transfer (FRET) processes, but also in solid-state lighting, solar energy harvesting, and information and quantum-information technologies. In particular the latter requires detailed knowledge of the quantum properties of the exciton quasiparticle including its wave-function in real as well as momentum $(k)$-space. In addition, exciton formation, relaxation and decay dynamics as well as their interaction with the solid or free charge carriers are of particular interest since those are, e.g., sources of decoherence hampering applications in the quantum information and computation sector.

Excitons can have finite crystal momentum with electrons and holes confined at different valleys in $k$ space. Exciton energies and the dynamics of excitons with vanishing center of mass momentum can be easily determined by (time-resolved) optical interband measurements. Binding and Rydberg energies can be determined either by absorption experiments or a combination of optical experiments and scanning-tunneling experiments and even the inner structure, independent of their center of mass momentum, can be accessed by time-resolved intraband spectroscopy . Direct experimental access to spin-forbidden or momentum dark excitons is more challenging because optical interband transitions need to be mediated, e.g., by phonons to fulfill the momentum conservation law. Still, the wavefunction properties and the dispersion relation $\mathrm{E}(k)$ of those quasiparticles describing the neutral excitation cannot be unraveled by such techniques. The dispersion relation of the underlying single-particle states can be accessed in the case of occupied states by angle-resolved photo-emission spectroscopy (ARPES) and in the case of unoccupied states by inverse photoemission experiments (IPE) .

Excitons like other quasiparticles possess a quantum mechanical wavefunction as well as energy-momentum relation that are distinctly different from those of the underlying single particle states. The concept of a quasiparticle is a great success story significantly simplifying the understanding and description of complex many-body phenomena. However, direct measurement of the spatial distribution of the quasiparticle wavefunction [Dong21] and direct visualization of finite momentum excitons [Dong21, remains challenging and was achieved only more than 90 years after the quasi-particle invention. Dong and coworkers [Dong21] employed a multi-dimensional photoemission spectroscopy scheme to directly access the time-, momentum-, and energy-resolved properties of excitons in the layered semiconductor $\mathrm{WSe}_{2}$. The decay dynamics of bright excitons was monitored through time-resolved and angle-resolved photoemission spectroscopy (tr-ARPES) measurements. The material is first excited with a pump pulse of energy close to the fundamental band gap and probed with a time-delayed XUV probe pulse to study the photoemission characteristics in dependence of the excitation in the energy and time domain. Near-resonant excitation conditions are fulfilled if the energy of the absorbed photon is just sufficient to promote an electron from the valence band at the $K$ -point in the $1^{\text {st }}$ Brillouin Zone (BZ) to the conduction band at the $K$-point leaving a hole behind. The $K$ -points are high symmetry point located at the corners of the $1^{\text {st }}$ BZ with the lowest energy direct interband transition in the band structure. For near resonance excitation, the generated particles have vanishing of just little access energy. For such a near-resonant excitation scheme, Dong et al. report the generation of a coherent excitonic polarization. The neutral elementary excitations decay to a bright exciton population within a dephasing time of about $17 \mathrm{fs}$. Rather fast within a time of about $18 \mathrm{fs}$ the quasiparticles scatter into an exciton ensemble with finite center of mass momentum with the electron state residing at another high symmetry point - the $\Sigma$ point inside the BZ and the hole state at the $K$-point at the corner of the BZ. By Fourier transformation of the momentum distribution, real space information of the excitonic state could 
be extracted [Dong21]. In this way, the isotropic quasiparticle wave function envelope is determined and an exciton Bohr radius of $1.78 \mathrm{~nm}$ is obtained, which is consistent with the theoretically expected valued.

In the future, the method of Dong et al. holds the promise to also uncover the spatial distribution of the quasiparticle wavefunction along with the distinct properties of other many-body states of interest. Among these are charge-transfer excitons. Due to reduced overlap of the underlying single-particle wave-functions, charge-transfer excitons exhibit enhanced lifetimes that allow the formation of thermalized dense ensembles. Such ensembles of bosonic quasiparticles are expected to show emergent correlation phenomena at high densities and low temperatures due to their bosonic nature. Valley selective hybridization effects enable electric-field and hence gate-tunable switching of charge excitons and direct excitons that might serve as quasiparticle qubits. For integration into realistic quantum-circuitries, it would be highly beneficial to know their quantum mechanical properties. The extension of the method introduced by Dong et al. [Dong21] can thus be expected to advance our current understanding of interaction-driven emergent phenomena in solid state systems.

[Dong21] 